\title{
Correlation of Typical Absorber Lined and Reverberation Chamber Compliance Measurements - How Likely?
}

\author{
Gustav J. Freyer \\ Consultant \\ Monument, $\mathrm{CO}$, USA
}

\author{
Mats G. Bäckström \\ Swedish Defence Research \\ Agency, FOI \\ PO Box 1165, S-581 11 \\ Linköping, Sweden \\ mats@foi.com
}

\author{
Michael O. Hatfield \\ Naval Surface Warfare Center, \\ Dahlgren Division \\ Dahlgren, VA USA \\ hatfieldmo@nswc.navy.mil
}

\begin{abstract}
Several EMC emission and immunity compliance standards specify a reference technique and acceptable alternative techniques. Generally, a correlation of failures from an alternative technique to the reference technique is required.

This work investigates the frequency-by-frequency correlation of typical absorber lined and reverberation chamber compliance tests. The investigation is based on extensive absorber lined and reverberation chamber data bases for coupling to instrumented test articles.

The results suggest the potential uncertainty in a frequency-by-frequency correlation of absorber lined and reverberation chamber tests.

\section{INTRODUCTION}

It is common, and in some cases required, to "correlate" results from alternative EMC compliance tests. In particular, it is desirable to compare failure data as a function of frequency from alternative techniques. The intrinsic problem for correlation attempts is that different test techniques measure different attributes of the equipment response.
\end{abstract}

In an absorber lined chamber (ALC) emission test the maximum electric field from two polarizations at one or more aspect angles around the equipment-under-test (EUT) is reported. A reverberation chamber (RC) emission test reports the radiated power from the EUT.

In an ALC immunity test the maximum EUT response to a specified electric field from two polarizations at one or more aspect angles is reported. An RC immunity test reports the EUT response to a statistically isotropic electromagnetic environment (EME) at a specified field equivalence level.

The effects of a limited aspect angle field measurement can be related to the effects of an isotropic measurement through the equipment response pattern. If the equipment response patterns are known, theory predicts and measurements on at least one research test article demonstrate, that the results of alternative tests can be correlated. [1]
Unfortunately equipment response patterns are not generally known a priori. The patterns can be complex and highly variable with frequency $[2,3]$. These factors suggest difficulty in attempting correlation between alternative compliance tests.

For complex equipment response structures, a limited ALC test is likely to miss the maximum response. The probability of missing the maximum is related to the directivity. For an RC measurement, the statistically isotropic EME produces a measurement offset from the maximum that is directly proportional to the directivity. Note that while the directivity is generally not known, recent work suggests that it can be estimated theoretically.[4] For test equipment whose failures are safety or mission critical, the maximum equipment response can be simulated by appropriately adjusting the RC test levels.

In previous work, the authors have shown that for measurements at different frequencies but with the same directivity, there is statistical correlation between limited aspect angle ALC and RC measurements. [5] An explanation is suggested through the randomization of the ALC offset from the maximum as the response pattern fine structure and its orientation changes with frequency. Unfortunately the statistical correlation does not necessarily imply good frequency-by-frequency correlation.

\begin{abstract}
APPROACH
As in other reported work by the authors the coupling cross section, or transfer function (TF), defined by the power received by an internal probe normalized by the power density of the incident field will be used in this investigation. $[6,7]$ The TF can be used as a surrogate for emissions, immunity, and shielding effectiveness tests.
\end{abstract}

The $\mathrm{TF}$ is available for at least 1400 frequencies covering $0.5-18 \mathrm{GHz}$ for two instrumented test articles for both ALC and RC tests. Both test articles have two internal 
probes. The dimensions are $33 \times 32 \times 25 \mathrm{~cm}$ and $30 \times 25 \times 8 \mathrm{~cm}$ for test articles 1 and 2 respectively. Both had unterminated external cables.

The ALC data considered were the TFs $\left(\mathrm{TF}_{\mathrm{ALC}}\right)$ to both probes for two polarizations of the six aspect angles normal to the EUT faces for a frequency range $0.5-18 \mathrm{GHz}$.

The RC data considered were the average TFs $\left.\left(<\mathrm{TF}_{\mathrm{RC}}\right\rangle\right)$ for each probe over the frequency range $0.5-18 \mathrm{GHz}$.

Correlation between the ALC and RC data will be based on a frequency dependent correlation factor $(\mathrm{CF}(\mathrm{f}))$. The $\mathrm{CF}(\mathrm{f})$ is defined as the ratio $\left\langle\mathrm{TF}_{\mathrm{RC}} \text { (f) }\right\rangle_{\text {measured }} /$ $\left\langle\mathrm{TF}_{\mathrm{RC}}(\mathrm{f})\right\rangle_{\text {predicted. }}$ The $\left\langle\mathrm{TF}_{\mathrm{RC}}(\mathrm{f})\right\rangle_{\text {predicted }}$ can be determined from face normal $\mathrm{TF}_{\mathrm{ALC}}$ measurements and an assumed equipment response pattern.

Following correlation attempts between alternative techniques (e.g., OATS - ALC, ALC - GTEM), this paper will consider the viability of simple equipment response pattern assumptions. The predicted and measured $\left\langle\mathrm{TF}_{\mathrm{RC}}\right\rangle$ are compared to evaluate the reasonableness of a pattern assumption as a correlation algorithm and to determine the range of uncertainty in the correlation.

\section{RESULTS}

For probe A of test article 1, the maximum of the two polarization $\mathrm{TF}_{\mathrm{ALC}}$ for each of the six faces is shown in Figure 1. Each marker represents a different face. For clarity in presentation, every sixth frequency is displayed over the range $0.5-18 \mathrm{GHz}$.

The intent of the Figure is to show the variability of the responses and the overall structure as functions of frequency. The range of face responses (maximum to minimum) at a given frequency varies from 2.7 to $30 \mathrm{~dB}$. This indicates the possible variation in test results for a limited aspect angle ALC test. Figure 1 is representative of the behavior of both probes of the two test articles.

Figure 1 also suggests the lack of symmetry in the equipment response pattern. A small spread in face response indicates a reasonably symmetric pattern. A large variation suggests an equipment response with little or no symmetry. The behavior shown in Figure 1 implies that an assumed pattern with a high degree of symmetry, as is frequently used in correlation attempts between alternative techniques, is unlikely to provide a good correlation algorithm.

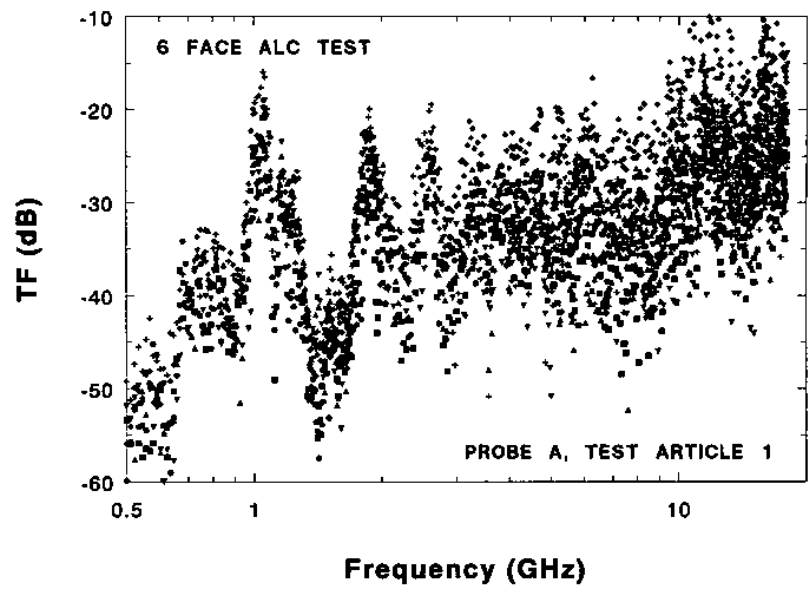

Figure 1. Maximum Absorber Lined Chamber Transfer Function for Two Polarizations for Six Faces for Probe A of Test Article 1.

Figure 2 shows the maximum $\mathrm{TF}_{\mathrm{ALC}}$ for probe $\mathrm{A}$ of test article 1 for a two-polarization test normal to face 1 . This is the face that engineering judgment would define as the reference face.

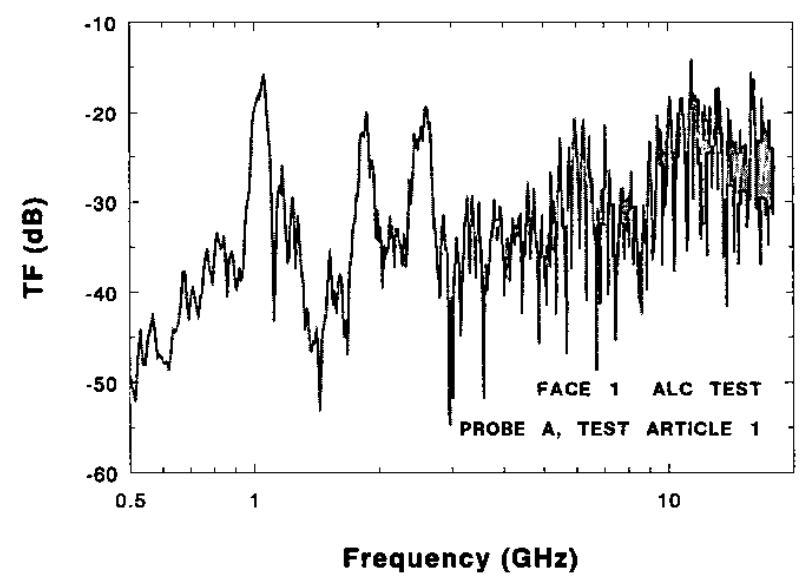

Figure 2. Maximum Absorber Lined Chamber Transfer Function for Two Polarizations for Face 1 for Probe A of Test Article 1.

A detailed analysis of Figures 1 and 2 show that face 1 provides the maximum response for most, but not all, of the frequencies up to about $2.5 \mathrm{GHz}$. Above $2.5 \mathrm{GHz}$ face 1 almost never has the maximum response.

The high probability that a single face contains the maximum response over a wide frequency interval $(0.5$ to 2.5 $\mathrm{GHz}$ in this case) is not observed for the other probe meas- 
urements (i.e. probe B of test article 1 and probes A and B of test article 2 ). These data show greater variability with frequency. Thus over the frequency interval $0.5-2.5$ $\mathrm{GHz}$, Figure 2 is a "best case".

Another feature of the response shown in Figures 1 and 2 is the apparent resonance coupling effects. Due to the logarithmic scale of these Figures the structures above about 3 $\mathrm{GHz}$, although present, are not apparent.

Figure 3 shows the $\left\langle\mathrm{TF}_{\mathrm{RC}}\right\rangle$ for probe $\mathrm{A}$ of test article 1 .

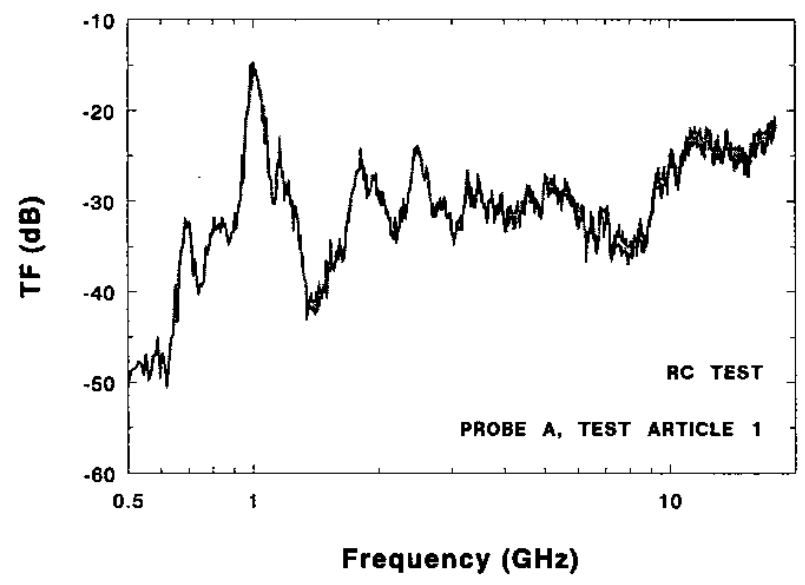

Figure 3. Reverberation Chamber Transfer Function for Probe A of Test Article 1.

A comparison of Figures 2 and 3 shows general trend agreement but significant variation at individual frequencies.

The CF is based on $\left\langle\mathrm{TF}_{\mathrm{RC}}\right\rangle_{\text {predicted }}$ from measured data and an assumed equipment response pattern. The first correlation algorithm considered is an isotropic response pattern although the data in Figure 1 does not support this assumption. In this case $\left\langle\mathrm{TF}_{\mathrm{RC}}\right\rangle_{\text {predicted }}=\mathrm{TF}_{\mathrm{ALC}}$. Figure 4 shows $\left\langle\mathrm{TF}_{\mathrm{RC}}\right\rangle_{\text {measured }} /\left\langle\mathrm{TF}_{\mathrm{RC}}\right\rangle_{\text {predicted }}$ for Face $1 \mathrm{ALC}$ measurements.

The $\left\langle\mathrm{TF}_{\mathrm{RC}}\right\rangle_{\text {measured }}$ is based on 200 tuner positions. The statistical estimate for the $99 \%$ confidence interval uncertainty in $\left\langle\mathrm{TF}_{\mathrm{RC}}\right\rangle_{\text {measured }}$ is $\pm 1.2 \mathrm{~dB}$. Therefore the spread in the CF is dominated by the ALC Face 1 results. A typical RC test using 12 tuner positions would have a larger uncertainty at each frequency yielding a larger spread in the $\mathrm{CF}$.

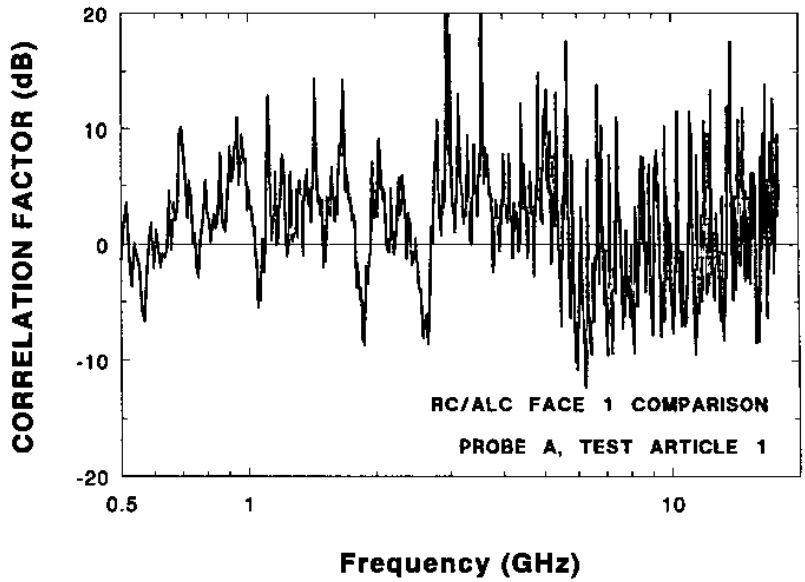

Figure 4. Correlation Factor for Reverberation Chamber Transfer Function and Two Polarization, Face 1 Absorber Lined Chamber Transfer Function for Probe A of Test Article 1.

Even though an isotropic response pattern is a poor description of the measured response pattern, the average $\mathrm{CF}$ in Figure 4 is $1.3 \mathrm{~dB}$. This suggests good statistical agreement between the alternative test techniques. As noted earlier, the authors have previously demonstrated that when results are averaged over many frequencies, the statistical equipment response error, that is the departure from the maximum equipment response measured in a robust test (many aspect angle ALC test) is comparable for both ALC and RC tests. [5]

Unfortunately, the statistical agreement is not applicable to the frequency-by-frequency correlation of concern in this paper. As an indication of the correlation problem, the range of the $\mathrm{CF}(\mathrm{f})$ (maximum to minimum) is $35 \mathrm{~dB}$. This is a potential problem for comparing compliance test failures between the two techniques.

A single aspect angle test of another face would show a different $C F$ but the frequency-by-frequency correlation does not improve. Since a single aspect angle ALC test has the potential for large uncertainties, some standards require a minimum of two polarization tests at four face normals. Using the plane which engineering judgment would define as the reference plane for a typical test setup, an analysis of the equivalent of Figures 2 and 4 was performed. Figure 5 shows the result of a two-polarization test at normals to the four faces of the reference plane.

The four-aspect angle ALC response is always equal to or greater than the one aspect angle response. Therefore the $\mathrm{CF}(\mathrm{f})$ as defined will show that the plane data is a lower bound on the face data. The maximum of a four-aspect angle, two polarization test yields a CF average of $-2.3 \mathrm{~dB}$ 
and reduces the $\mathrm{CF}$ range to $29 \mathrm{~dB}$. But note the frequency-by-frequency correlation does not improve significantly.

The two untested faces (not in the test plane) dominate the response above about $3 \mathrm{GHz}$. As a result, correlation attempts for a six aspect angle test would be significantly worse than indicated in Figure 4 and 5 for frequencies above about $3 \mathrm{GHz}$. This result is somewhat counterintuitive but it can be explained by relatively high directivities centered on the two untested faces.

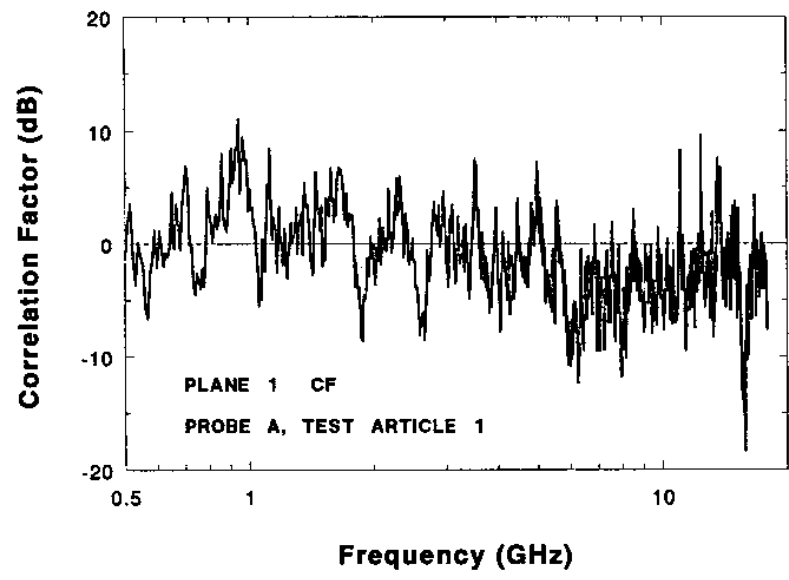

Figure 5. Correlation Factor for Reverberation Chamber Transfer Function and Two Polarization, Plane 1 Absorber Lined Chamber Transfer Function for Probe A of Test Article 1.

Typical correlation attempts assume that equipment response patterns can be approximated by simple structures like azimuthally symmetric dipoles. Assuming a quarter wave dipole oriented with its symmetry axes perpendicular to the reference plane, the CF would be offset by a constant factor of $2.2 \mathrm{~dB}$ over the entire frequency interval. Figures 4 and 5 indicate this would not improve the frequency-byfrequency correlation. Generalizing this result, it can be seen that no fixed orientation, azimuthally symmetric pattern, even a frequency dependent pattern, will yield an improvement in the frequency-by-frequency correlation.

The results for the other three probes, while different in detail, show the same issues.

Figure 6 shows the cumulative distribution of the $0.5-18$ $\mathrm{GHz}$ data of Figure 4. This presentation permits an assessment of the probability that the frequency-by-frequency CF will be within specified limits.
For example, the markers indicate that there is a $34 \%$ probability that the $\mathrm{CF}$ will be within $\pm 2.5 \mathrm{~dB}$. The probability of any desired uncertainty interval can be determined from Figure 6.

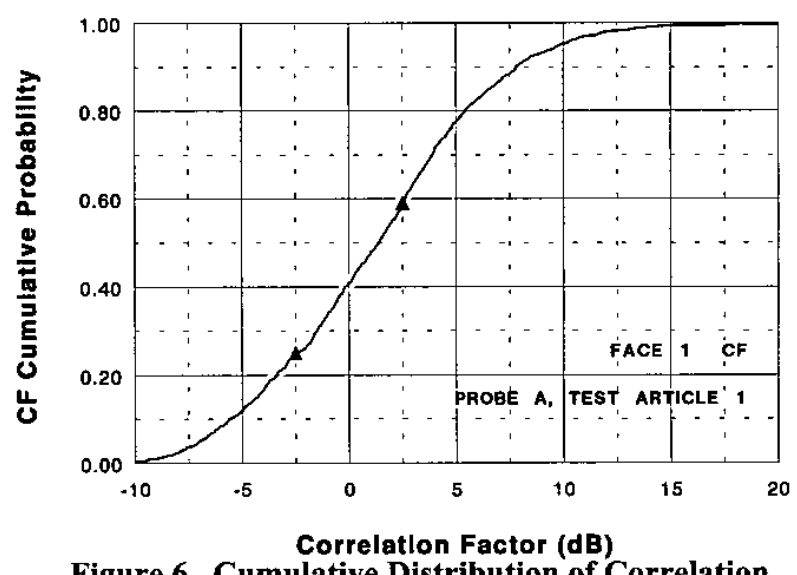

Figure 6. Cumulative Distribution of Correlation Factor for Reverberation Chamber Transfer Function and Two Polarization, Face 1 Absorber Lined Chamber Transfer Function for Probe A of Test Article 1.

Representative $\mathrm{CF}$ parameters are provided in Tables 1,2 , and 3 for probe $A$ of test article 1 and probes $A$ and $B$ of test article 2 respectively for different frequency bands.

Table 1. Statistical Parameters for the Correlation Factor between Reverberation Chamber and Single Aspect Angle, Two Polarization Absorber Lined Chamber Tests of Face 1 for Probe A of Test Article 1.

\begin{tabular}{|c|c|c|c|}
\hline $\begin{array}{c}\text { Freq } \\
\text { Band } \\
(\mathrm{GHz})\end{array}$ & $\begin{array}{c}\text { Range } \\
\mathrm{dB}\end{array}$ & $\begin{array}{c}\text { Prob } \\
\mathrm{CF}> \pm 2.5 \mathrm{~dB}\end{array}$ & $\begin{array}{c}\text { Prob } \\
\mathrm{CF}> \pm 5 \mathrm{~dB}\end{array}$ \\
\hline $0.5-1.0$ & 18 & $40 \%$ & $28 \%$ \\
\hline $1.0-2.0$ & 23 & $68 \%$ & $30 \%$ \\
\hline $2.0-4.0$ & 32 & $67 \%$ & $36 \%$ \\
\hline $0.5-18$ & 35 & $66 \%$ & $34 \%$ \\
\hline
\end{tabular}

As indicated earlier, over the frequency interval $0.5-2.5$ $\mathrm{GHz}$, these data represent a "best case" for probe A of test article 1 . 
Table 2. Statistical Parameters for the Correlation Factor between Reverberation Chamber and Single Aspect Angle, Two Polarization Absorber Lined Chamber Tests of Face 1 for Probe A of Test Article 2.

\begin{tabular}{|c|c|c|c|}
\hline $\begin{array}{c}\text { Freq } \\
\text { Band } \\
(\mathrm{GHz})\end{array}$ & $\begin{array}{c}\text { Range } \\
\mathrm{dB}\end{array}$ & $\begin{array}{c}\text { Prob } \\
\mathrm{CF}> \pm 2.5 \mathrm{~dB}\end{array}$ & $\begin{array}{c}\text { Probability } \\
\mathrm{CF}> \pm 5 \mathrm{~dB}\end{array}$ \\
\hline $0.5-1.0$ & 22 & $53 \%$ & $14 \%$ \\
\hline $1.0-2.0$ & 13 & $57 \%$ & $26 \%$ \\
\hline $2.0-4.0$ & 22 & $65 \%$ & $40 \%$ \\
\hline $0.5-18$ & 44 & $86 \%$ & $74 \%$ \\
\hline
\end{tabular}

Table 3. Statistical Parameters for the Correlation Factor between Reverberation Chamber and Single Aspect Angle, Two Polarization Absorber Lined Chamber Tests of Face 1 for Probe B of Test Article 2.

\begin{tabular}{|c|c|c|c|}
\hline $\begin{array}{c}\text { Freq } \\
\text { Band } \\
(\mathrm{GHz})\end{array}$ & $\begin{array}{c}\text { Range } \\
\mathrm{dB}\end{array}$ & $\begin{array}{c}\text { Prob } \\
\mathrm{CF}> \pm 2.5 \mathrm{~dB}\end{array}$ & $\begin{array}{c}\text { Prob } \\
\mathrm{CF}> \pm 5 \mathrm{~dB}\end{array}$ \\
\hline $0.5-1.0$ & 17 & $46 \%$ & $35 \%$ \\
\hline $1.0-2.0$ & 16 & $94 \%$ & $73 \%$ \\
\hline $2.0-4.0$ & 23 & $83 \%$ & $62 \%$ \\
\hline $0.5-18$ & 30 & $76 \%$ & $50 \%$ \\
\hline
\end{tabular}

In the tables the range indicates the maximum to minimum $\mathrm{CF}$ in each of the four frequency bands. The majority of values are greater than $20 \mathrm{~dB}$. Also indicated are the probabilities that the frequency-by-frequency $\mathrm{CF}$ will be $> \pm$ $2.5 \mathrm{~dB}$ and $> \pm 5 \mathrm{~dB}$. Note that for the three data sets the probability that the $\mathrm{CF}(\mathrm{f})$ exceeds $\pm 5 \mathrm{~dB}$ is generally greater than $25 \%$.

Tables 2 and 3 indicate the substantial differences between the CFs for two probes in the same test article. This suggest a potential effect on correlation due to internal spatial variability of dominant response functions.

\section{CONCLUSIONS}

This paper investigated the correlation between ALC and $\mathrm{RC}$ measurements on a frequency-by-frequency basis for an extensive data base of coupling to instrumented electronic equipment over a frequency interval of $0.5-18$ $\mathrm{GHz}$. The coupling can be considered a surrogate for either emissions, immunity, or shielding effectiveness testing.
The ALC measurements on the two test articles indicate the coupling to an internal probe varies significantly with aspect angle. This implies a highly structured equipment response pattern. The pattern can also have substantial changes over small changes in frequency. The variation in coupling convoluted with the internal electronic emission or immunity characteristics yields a pass-fail profile as a function of frequency. In particular these results suggest the pass-fail profiles may vary significantly with the ALC test aspect angles. This effect will impact correlation attempts between ALC and RC tests.

The frequency-by-frequency correlation between ALC and $\mathrm{RC}$ tests was evaluated in terms of a correlation factor defined as $\left\langle\mathrm{TF}_{\mathrm{RC}}(\mathrm{f})\right\rangle_{\text {measured }} /\left\langle\mathrm{TF}_{\mathrm{RC}}(\mathrm{f})\right\rangle_{\text {predicted. }}$ The $<\mathrm{TF}_{\mathrm{RC}}$ (f) $\rangle_{\text {predicted }}$ was determined from the ALC measurements and an assumed isotropic equipment response pattern. This pattern, while not physically justified at anyone frequency, apparently performs a pattern averaging over frequency. It was suggested that other simple symmetric response patterns are also not supported by the ALC measurements. Further they will introduce arbitrary and equally unsupported offsets in the correlation factor.

In summary, this investigation suggests that uncertainties in frequency-by-frequency correlation between absorber lined chamber and reverberation chamber measurements may have a standard deviation on the order of $5 \mathrm{~dB}$ and a maximum to minimum range of more than $20 \mathrm{~dB}$.

The reader can decide whether uncertainties of this magnitude are acceptable for correlation equipment failure levels between absorber lined and reverberation chambers.

\section{ACKNOWLEDGMENTS}

The authors acknowledge the support of the US Naval Surface Warfare Center, Dahlgren Division, the Swedish Armed Forces and the Swedish Defence Material Administration.

\section{REFERENCES}

[1] Koepke, G., "Emissions Measurements in a Reverberation Chamber, OATS, and Anechoic Chamber", The 1999 Mode-Stirred Chamber, Anechoic Chamber and OATS Users Meeting, Northbrook, IL, June 1999

[2] Freyer. G.J., and M.G. Bäckström, "Some Implications of a Single Aspect Angle Electromagnetic Compatibility Test", 19th Digital Avionics System Conference, Philadelphia, PA, Oct 2000 . 
[3] Freyer, G.J. "Implications of Test Article Response Patterns for EMC Testing", 2001 Reverberation Chamber, Anechoic Chamber and OATS Users Meeting, Bellevue, WA, June 2001.

[4] Koepke, G., Hill, D., and J. Ladbury, "Directivity of the Test Device in EMC Measurements", IEEE Int. Symposium on EMC, Washington, DC, August 2000.

[5] Freyer, G.J., and M.G. Bäckström, "Comparison of Anechoic and Reverberation Chamber Coupling Data as a Function of Directivity Pattern - Part II", IEEE Int. Symposium on EMC, Montreal, Canada, August 2001.
[6] Jansson, L., and M.G. Bäckström, "Directivity of Equipment and its Effect on Testing in Mode-Stirred and Anechoic Chamber", IEEE Int. Symposium on EMC, Seattle, WA, August 1999.

[7] Freyer, G.J., and M.G. Bäckström, "Comparison of Anechoic \& Reverberation Chamber Coupling Data as a Function of Directivity Pattern", IEEE Int. Symposium on EMC, Washington, DC, August 2000. 